\title{
A Disturbance Observer Based Backstepping Controller Design for Missile Pitch Channel Systems
}

\author{
Wenguang Zhang ${ }^{\mathrm{a}}$, Lili Yang, Junwei Lei
}

Department of Control, Naval aeronautical and astronautical university, Yantai, 264001,China

aemail: zhangwenguang1024@126.com

Keywords: Disturbance; Observer; Backstepping; Missile; Control system

\begin{abstract}
A kind of disturbance observer method was adopted in backstepping controller design of pitch channel missile control system. Not only the outer disturbance but also the inner uncertainties of missile model can be estimated by two disturbance observer. Also the stability of the whole system was proved by constructing a Lyapunov function which is separate and not related with the stability of the observer. At last, detailed numerical simulation was done with a real simplified pitch channel system model to testify the effectiveness of the double observers backstepping control.
\end{abstract}

\section{Introduction}

Disturbance observer method [1-4] was widely used in missile system and controller design since not only the real disturbance can be solved by observer, but also system uncertainties can be viewed as a kind of outer disturbance[5-9]. A kind of accurate differentiator can also be used as a kind of disturbance observer. But disturbance observer is effective[10-14] for first order systems, so second order simplified missile system need to design two disturbance observers. In this paper, a second order missile system was divided into two first order systems, and a special structure with backstepping and observers was constructed to solve the attack angle tracking problem. Design and simulation result shows that the proposed method is very effective.

\section{Problem Description}

The simplified linear model of supersonic missile pitch channel with disturbance can be written as following second order system:

$$
\begin{aligned}
& \dot{\alpha}=\omega_{z}-a_{34} \alpha-a_{35} \delta_{z}+f_{1} \\
& \dot{\omega}_{z}=a_{24} \alpha+a_{22} \omega_{z}+a_{25} \delta_{z}+f_{2}
\end{aligned}
$$

Where $a_{i j}$ is air dynamic coefficient of missile, $\alpha$ is attack angle of missile, $\omega_{z}$ is the rotate speed of pitch angle, $f_{1}$ and $f_{2}$ are outer disturbance.

The control objective is to design a control law $\delta_{z}$ such that the attack angle $\alpha$ can track the desired angle $\alpha^{d}$. Without loss of generality, assume $\alpha^{d}=1$, and the disturbance $f_{1}$ and $f_{2}$ can be observed and compensated by the designed control law $\delta_{z}$.

\section{Disturbance Observer Design}

For the first subsystem, we assume the following part $-a_{35} \delta_{z}+f_{1}$ as disturbance $D$, then it satisfies

$$
-a_{35} \delta_{z}+f_{1}=D
$$

And the above system can be rewritten as.

$$
\dot{\alpha}=\omega_{z}-a_{34} \alpha+D
$$

And we construct a disturbance observer as 


$$
\begin{aligned}
& \dot{\hat{\alpha}}=\omega_{z}-a_{34} \alpha+\hat{D} \\
& \hat{D}=-k_{0}|\hat{\alpha}-\alpha|^{1 / 2} \operatorname{sign}(\hat{\alpha}-\alpha)+\hat{d} \\
& \dot{\hat{d}}=-k_{1} \operatorname{sign}(\hat{d}-\hat{D})
\end{aligned}
$$

Where $\hat{d}$ and $\hat{D}$ are the estimations of disturbance $D, \hat{\alpha}$ is the estimation of state $\alpha$.

\section{Backstepping Controller Design}

Define a new error variable as $e_{\alpha}=\alpha-\alpha^{d}$, then the first subsystem can be described as

$$
\dot{e}_{\alpha}=\omega_{z}-a_{34} \alpha+D-\dot{\alpha}_{d}
$$

Then the idea value of $\omega_{z}$ can be designed as $\omega_{z}^{d}$ as follows:

$$
\omega_{z}^{d}=a_{34} \alpha-\hat{D}+\dot{\alpha}_{d}-k_{\alpha_{1}} e_{\alpha}-k_{\alpha 2} \frac{e_{\alpha}}{\left|e_{\alpha}\right|+\varepsilon_{b}}
$$

And Define a new variable as $e_{\omega}=\omega-\omega_{z}^{d}$, then the second subsystem can be rewritten as

$$
\dot{e}_{\omega}=a_{24} \alpha+a_{22} \omega_{z}+a_{25} \delta_{z}+f_{2}-\dot{\omega}_{z}^{d}
$$

And we assume $f_{2}-\dot{\omega}_{z}^{d}$ as another disturbance $W$, then it has $W=f_{2}-\dot{\omega}_{z}^{d}$, and use another disturbance observer to estimate it, then a disturbance observer can be constructed as

$$
\begin{aligned}
& \dot{\hat{e}}_{\omega}=a_{24} \alpha+a_{22} \omega_{z}+a_{25} \delta_{z}+\hat{W} \\
& \hat{W}=-k_{w 0}\left|\hat{e}_{\omega}-e_{\omega}\right|^{1 / 2} \operatorname{sign}\left(\hat{e}_{\omega}-e_{\omega}\right)+\hat{w} \\
& \dot{\hat{w}}=-k_{w 1} \operatorname{sign}(\hat{w}-\hat{W})
\end{aligned}
$$

Then the real control law can be designed as

$$
\delta_{z}=\frac{1}{a_{25}}\left\{-a_{24} \alpha-a_{22} \omega_{z}-k_{a 1} e_{\omega}-k_{a 2} \frac{e_{\omega}}{\left|e_{\omega}\right|+\varepsilon_{a}}-\hat{W}\right\}
$$

Then if we choose a Lyapunov function as

$$
V=\frac{1}{2} e_{\alpha}^{2}+\frac{1}{2} e_{\omega}^{2}
$$

And its derivative can be calculated as

$$
\dot{V}=-k_{\alpha 1} e_{\alpha}^{2}-k_{\alpha 2} \frac{e_{\alpha}^{2}}{\left|e_{\alpha}\right|+\varepsilon_{b}}-k_{a 1} e_{\omega}^{2}-k_{a 2} \frac{e_{\omega}^{2}}{\left|e_{\omega}\right|+\varepsilon_{a}}+e_{\alpha} \tilde{D}+e_{\omega} \tilde{W}
$$

Where error of disturbance observer $\tilde{D}$ and $\tilde{W}$ is defined as

$$
\tilde{D}=D-\hat{D}, \tilde{W}=W-\hat{W}
$$

Obviously, if the error of disturbance observer is small enough, then it satisfies

$$
\dot{V} \leq-k_{\alpha 1} e_{\alpha}^{2}-k_{a 1} e_{\omega}^{2}
$$

And according to the design of observer method, we can choose big enough gains such that the error of observer is small enough. So according to the Lyapunov stability theorem, the whole system is stable.

\section{Numerical Simulations}

The air coefficient of a type of missiles can be set as

$$
a_{25}=-167.87 ; a_{35}=0.243 ; a_{22}=-2.876 ; a_{24}=-193.65 ; a_{34}=1.584
$$

It is a kind of non-mini-phase system. Set control parameters as

$$
k_{a 1}=8 ; k_{a 2}=15 ; k_{\alpha 1}=20 ; k_{\alpha 2}=10
$$

Then simulation results can see below figures 1-5. 


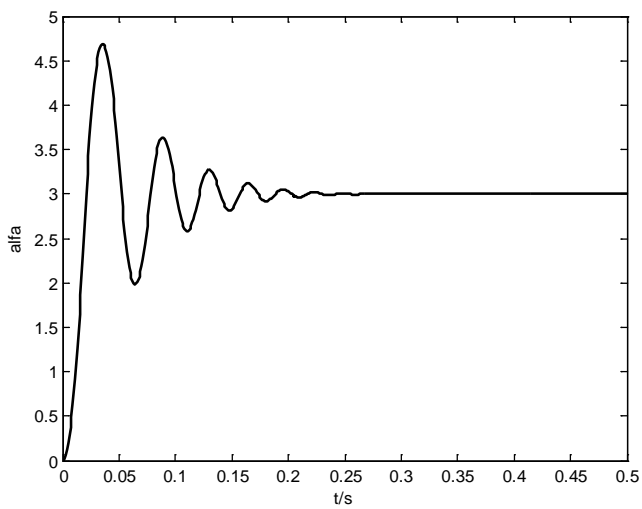

Figure 1 The curve of attack angle

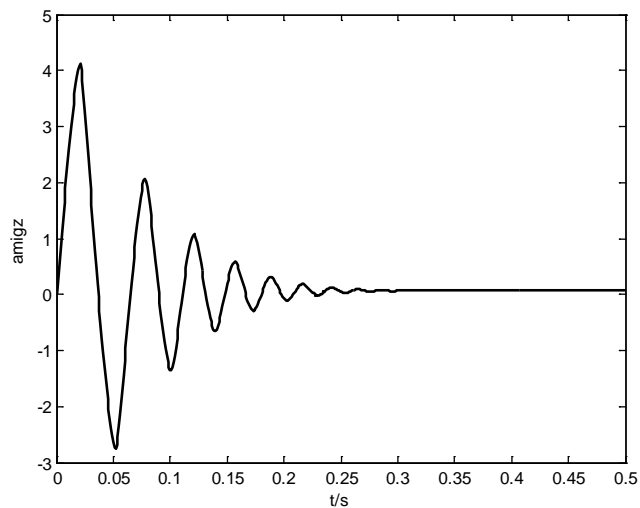

Figure 2 The figure of angle speed

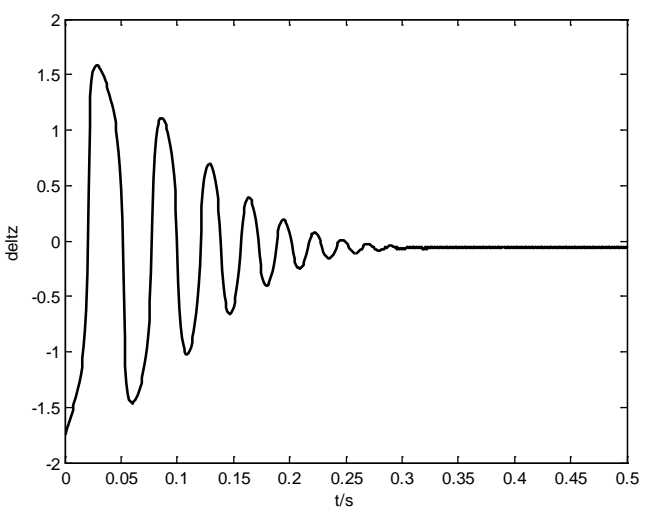

Figure 3 The curve of actuator

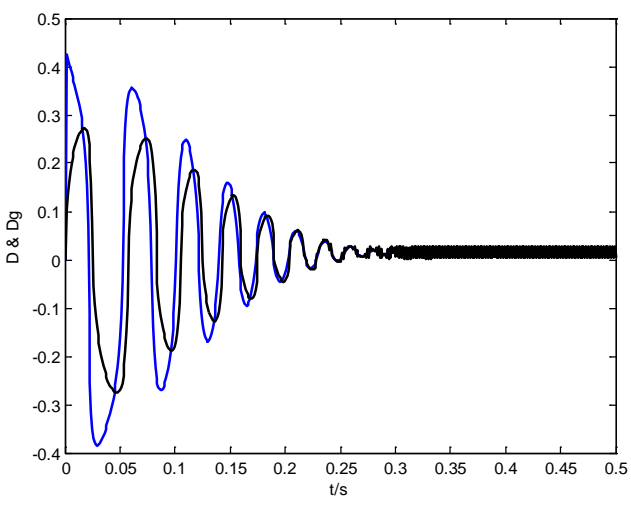

Figure 4 The estimation of disturbance D 


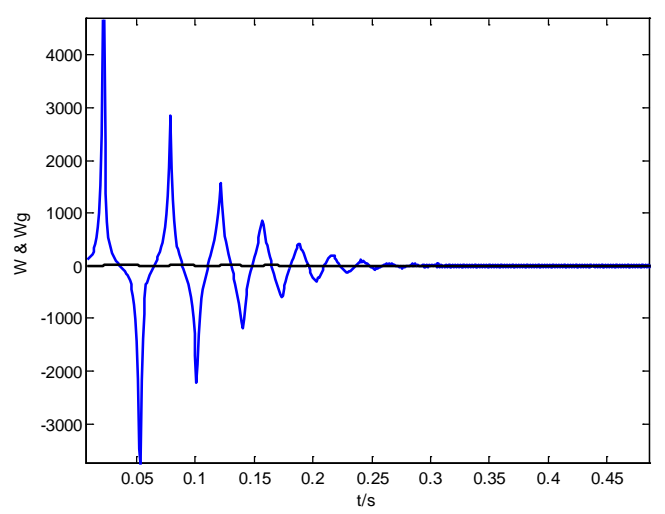

Figure 5 The estimation of disturbance $\mathrm{W}$

Figure 1 to Figure 3 shows the missile system is stable and attack angle can trace to the desired value 3 degree. And figure 4 and figure 5 shows that the disturbances can be observed and they can converge to the real value. So the whole design method is right and effective.

\section{Conclusions}

A novel attack angle tracking controller was designed by using disturbance observer method. And all uncertainties of pitch channel missile system can be solved by introducing two disturbance observer. And backstepping method then was used to guarantee the stability of the whole system. Also numerical simulation was done to testify the rightness of this new observer based missile control system design method.

\section{Reference}

[1] Feigenbaum M J.Quantitative universality for a class of nonlinear transformations [J], J.Stat. Phys. 1978, 19:25-52.

[2] Pecora L M and Carroll T L.Synchronization in chaotic systems[J],Phys.Rev. Lett.1990, 64:821-824.

[3] GE S S, Wang C, Lee T H. Adaptive backstepping control of a class of chaotic systems[J]. Int J Bifurcation and chaos. 2000, 10 (5): 1140-1156.

[4] GE S S, Wang C, Adaptive control of uncertain chus's circuits[J]. IEEE Trans Circuits System. 2000, 47(9): 1397-1402.

[5] Alexander L, Fradkov, Markov A Yu. Adaptive synchronization of chaotic systems based on speed gradient method and passification[J]. IEEE Trans Circuits System 1997,44(10):905-912.

[6] Dong X. Chen L. Adaptive control of the uncertain Duffing oscillator[J], Int J Bifurcation and chaos. 1997,7(7):1651-1658.

[7] Tao Yang, Chun-Mei Yang and Lin-Bao Yang, A Detailed Study of Adaptive Control of Chaotic Systems with Unknown Parameters[J] . Dynamics and Control. 1998,(8):255-267.

[8] M.T. Yassen, Chaos control of chaotic dynamical systems using backstepping design, Chaos Soliton Fract. 27 (2006) 537-548.

[9] Fengxiang Chen, Lin Chen, Weidong Zhang, Stabilization of parameters perturbation chaotic system via adaptive backstepping technique, Applied Mathematics and Computation 200 (2008) 101-109.

[10] M.T.Yassen, Adaptive chaos control and synchronization for uncertain new chaotic dynamical system, Physics Letters A 350 (2006) 36-43. 
[11] Jianping Yan, Changpin Li. On synchronization of three chaotic systems. Chaos, Solitons and fracrals. 2005,(23):1683-1688.

[12] Tournes, C., Landrum.D.B., Shtessel,Y., and Hawk,C.W., Ramjet-Powered Reusable Launch Vehicle Control by Sliding Modes, Journal of Guidance, Control and Dynamics,V01.2 1, No.3, 1998, PP.409-415

[13] Marrison, C. and Stengel R. Design of Robust Control Systems for a Hypersonic Aircraft, Jourmal of Guidance, Control and Dynamics,V01.21,No.1,1998,PP.58-63

[14] Wang,Q.,and Stengel,R.,Robust Nonlinear Control of a Hypersonic Aircraft, Jourmal of Guidance, Control and Dynamics,V01.23,No.4,2000,PP.577-585 